\title{
Hodgkin Lymphoma in Nigeria: Prevalence, Mechanisms of Tumorigenesis and Diagnosis
}

\section{Opara SC and Dokunmu TM*}

Department of Biochemistry, Covenant University, Nigeria

*Corresponding author: Titilope Modupe Dokunmu, Department of Biochemistry, Covenant University, Ota, Nigeria, Email: Titilope.dokunmu@covenantuniversity.edu.ng

\section{Mini Review \\ Volume 4 Issue 2}

Received Date: September 16, 2021

Published Date: November 02, 2021

DOI: $10.23880 /$ aabsc- 16000168

\section{Abstract}

Hodgkin lymphoma (HL) is an uncommon neoplasm where tumor stems from a certain kind of leukocytes known as B-cell. This lymphoid malignancy involves outlying lymph gland which may extend to tissues. Research estimates $40 \%$ of patients are diagnosed from constitutive signs while early detection is a challenge. The Hodgkin Reed-Stenberg cells is established as the hallmark of Hodgkin lymphoma but multiple underlying mechanisms are involved. Hodgkin lymphoma spreads across different regions with limitations of effective control, hence there is a need to fully understand its tumorigenesis and diagnosis. We reviewed the multiphase deregulated signalling pathways and several interactions amongst cells, these characteristics can proffer new breakthrough to target therapeutics either through direct inhibition of affected signalling pathways or Hodgkin Reed-Stenberg cells associated with diverse cellular infiltrates within the lymph cell. HL incidence in Nigeria is similar to the incidence in other regions but there is a higher death rate in Nigeria. Nigeria bears the second highest burden of HIV globally which contributes to incidence of HL. Also, Epstein-Barr virus increases the risk of the disease, which is also prevalent in Nigeria. Lymphomas are associated with higher cancer-related deaths especially in HIV-infected persons with 2.5times lower 2-year survival chance compared to HIV-uninfected persons and late stage of lymphoma was also a contributing factor to the survival outcomes. Hence, to reduce the burden of the disease, an understanding of the aetiology, epidemiology, and biochemical mechanisms of the lymphoma is needed which are herein reviewed.

Keywords: Hodgkin Lymphoma; Leukocytes; Aetiology; Epidemiology; Biochemical Mechanisms

Abbreviations: HL: Hodgkin Lymphoma; IM: Infectious Mononucleosis; EBV: Epstein Barr Virus; HRS: Hodgkin Reed Sternberg; LH: Lymphocytic Histiocytic.

\section{Introduction}

Lymph neoplasm consists of diverse malignant diseases, originating from lymphatic system [1]. The tissues there in are connective lymph cells that initiates a defence mechanism to pathogenic agents. These tissues look like tubes that carry lymphatic fluid through the lymphatic system and are also called lymphatic vessels. Leukocytes (white blood cells) and lymph (clear fluid) flow within the lymphatic vessel to the nodes where they exert their function as antibodies. Hodgkin lymphoma (HL) formerly identified as Hodgkin disease, is a kind of malignant tumor that stems from leukocytes known as called B- lymph cells [1]. It is an uncommon neoplasm of the lymph system which results in deregulation of the Bleukocytes leaving the sufferer highly prone to numerous infections. Lymphocytes are the main types of cells found in the lymph, HL is observed when (R-S cells) Reed-Sternberg is identified. R-S cells are big, wide and atypical lymph cell 
having either single or multiple nucleus. They can be either mononucleated, named Hodgkin cells or multinucleated, described as Reed-Sternberg cells [2,3].

Characteristic features of HRS cell within the lymphatic system tend to increase in size and extend to other tissues which includes lungs, liver, or bone marrow. Lymph tissues are located almost everywhere in human body hence increases the possibility of HL occurring wherever. According to WHO, HL is classified based on the identification of two histological forms: the nodular lymphocyte-predominant and the "classical" HL. The last mentioned is grouped into different types: nodular sclerosis, mixed cellularity, leukocyte depletion, and leukocyte-rich [4]. HL is characterized by the existence of huge multiphase nuclear Hodgkin Reed-Stenberg cells in the classic variation of Hodgkin lymphoma, while lymphocytic and histiocytic ( $\mathrm{L}$ and $\mathrm{H}$ ) cells are pathogenic for nodular LPHL. In the subclasses the neoplastic cells are bordered to extent of varying level by responsive non-cloned hematopoietic cells which gives rise to complicated web of organs affected by Hodgkin lymphoma. It is eventually proven that the signal pathway is two-directional, i.e. cells in the malignant lymph microenvironment both allow the existence of malignant cells which also modulates themselves by the neoplastic cells [5].

According to World Health Organization groupings, the histologic groups of Hodgkin lymphoma are nodule lymphocytic prevalent lymphoma and its occurrence is approximately five percent of entire Hodgkin lymphoma cases while classical lymphoma accounts for the rest Campo, et al. The classic lymphoma is further divided which includes: nodule induration, assorted cellularity, reduced lymph cell as well as rich lymph cell type [6].

- Nodular lymphocyte-predominant HL: This form of $\mathrm{cHL}$ is not associated with EBV, accounting 5\% of entire HL, occurs frequently in males when compared to females. Staging in nLPHL is estimated at $75 \%$ of patients in early stage of the lymphoma and possible tendencies of transforming into lethal level of nonHodgkin lymphoma.

- Nodular sclerosis cHL: It is associated with EBV at a relatively average level. Presents in about $10-40 \%$ of EBV-positive patients. It shows the incidence of cHL in Europe and North America, presenting Mediastinal mass in $80 \%$ patients and displays good future diagnostic predictions compared to other forms of $\mathrm{cHL}$.

- Mixed-cellularity cHL: It is strongly associated with EBV, presenting $75 \%$ of EBV-positive patients tested of cHL occurs frequently in HIV-positive patients and in underdeveloped nations. Frequent occurrence of adenopathy are of the peripheral and abdomen regions. Also $30 \%$ spleen infiltrates occur in patients.
- Leukocyte-rich cHL: Averagely associated with EBV, constitutes $5 \%$ of $\mathrm{cHL}$ prevalence of lymph adenopathy of the peripheral system is frequent and infrequent occurrence of mediastinal mass.

- Lymphocyte-depleted cHL: Strongly associated, with approximately $75 \%$ patients that are Epstein Barr virus seropositive, highly uncommon form of cHL estimated at less than 1\% occurrence North America and Europe. It occurs frequently with HIV infected patients in underdeveloped countries. Patients with this form of $\mathrm{cHL}$ are usually in the late stage of the lymphoma.

Precise grouping stages provided for the lymph malignancy is based on Ann Arbor staging system, established in a conference in 1971. This form of staging depicts positions involved and the occurrence of the tumor above and/or beneath the diaphragm. Stage I is localized disease, usually in a lymph node or tissue only, occur frequently at the neck region above the diaphragm, stage II occurs in more than two lymph nodes, same region and side of the diaphragm, stage III is similar to stage II but occurs beneath and above diaphragm while for stage IV, disease spreads or extends to multiple tissues with or without lymph nodes involved [7].

\section{Hodgkin Reed-Stenberg Cells and Variants}

Hodgkin Reed-Stenberg cells are identified as the basic neoplastic malignancy of HL which seldom affect the extranodal lymph organs. They are big, (30 $\geq 50 \mathrm{um})$ abnormal lymphocytes that may constitute multinucleate cells or duallobe bilobed nucleus with prominent eosinophilic inclusionlike nucleoli (like "owl's eye"). HRS cells are CD15 and 30 immunoreactive and are primary immunomarkers examining HL [2]. The diagnostic criteria include component of tumor cells that is Reed-Sternberg cell comprising of the exact and its various forms also it's responsive component such as the normal mature lymph cells, eosinophiles, plasmocytes, neutrophiles, fibrotic cells also capillaries. Histologic classes of Hodgkin's malignancy is based on the lymph nodes design, fraction identified amongst tumors, tumor less elements and morphological state of Reed-Sternberg cell and compositions of responsive infiltrates. These variants include:

- Hodgkin cell (a typical single nucleus RSC - It has similar features also a multinucleate cell.

- Lacunar RSC - big, contains one wild lobular nucleus, diverse little nucleoli and eosinophilic cytoplasm that retracts around the nucleus, creating an empty space ("lacunae").

- Pleomorphic RSC - consists of several inconsistent nuclei.

- Lympho-histiocytic ("pop-corn") variant - consists of little cell, highly lobated nucleus and little nucleoli.

- "Mummy" RSC-Characterized with dense nucleus, absent nucleolus and basophil in cytoplasm. 


\section{Global Prevalence of Hodgkin Lymphoma}

In 2020 , over 101,133 cases and over 32,560 deaths occurring from HL were reported globally by GLOBOCAN in 2018, and this malignancy can be cured in most cases [8]. The outstanding characteristic expression of this malignancy occurs in approximately $\leq 1 \%$ of neoplasm $[4,9]$. It is estimated that approximately $\leq 1$ of 25,000 people are affected by this cancer annually and accounts for about $1 \%$ of all cancers that occur in the world. According to IARC, GLOBOCAN (2012), the occurrence of Hodgkin lymphoma is 2.7-2.8 per 100,000 persons annually $(2.44 / 100,000$ persons in Nigeria), however this varies considerably by age, gender, ethnic groups, geographical regions and socio-economic status [10]. Also recent statistics of IARC, GLOBOCAN (2018) estimates 79,990 i.e. $0.4 \%$ incidence with mortality of 26,167 i.e. $0.3 \%$ cancer incidence globally [11]. There has been upsurge in the incidence trend of Hodgkin lymphoma. Studies from GDB reveals data on rise in rates of HL from 72,937 to 101,133 cases and mortality of 35,946 to 32,560 between 1990-2017 [10,11]. HL is an unusual neoplasm, with 7000-7500 new diagnosis yearly in the United States and United Kingdom and majority of the affected people are diagnosed at the early occurrence of the disease [4]. In Nigeria, about 2030 cases were diagnosed in 2020 and a 5-year prevalence of 5031 (GLOBOCAN - Nigeria Factsheet, 2020). Also in the Mediterranean basin populations such as France and Italy, incidence rates are estimated at 2.51 male to 2.74 female and 2.76 male to 2.02 female, respectively with mortality occurrence at 0.29 and 0.37 respectively [9], whereas incidence and mortality in Nigeria is estimated at $1.6 \%$ and $1.2 \%$, the mortality is about 4 times the risk of death in other regions (GLOBOCAN - Nigeria Factsheet, 2020). HL can affect individuals of any age, however, occurs frequently between two age groups specifically i.e. 15-35 and above 55 years respectively. Generally occurrence of lymphomas in Africa is very minimal including association of risk factors such as HIV [12]. HL incidence in Nigeria is similar to the incidence in other regions but there is a higher death rate in Nigeria, hence this review focuses on HL in Nigeria to review the clinical patterns, trends and contributory factors to the incidence of HL and how this can be overcome.

\section{Hodgkin Lymphoma in Nigeria}

We conducted a systematic search on PUBMED (www. pubmed.ncbi.nlm.nih.gov) of studies reporting Hodgkin lymphoma from Nigeria, using the search keywords: Hodgkin lymphoma AND Nigeria within title and abstract, the search returned 17 articles from which we reviewed 6 discussing Hodgkin lymphoma specifically. In 2020, HL ranked the 12th most common cancer in Nigeria with 2030 new cases and 919 deaths reported in 2020 (GLOBOCAN, 2021). We reviewed briefly other reported studies on incidence, clinicopathological features and frequency of occurrence of Hodgkin lymphoma. A 25 year review of cases seen at the University of Benin teaching hospital presents a dual-peak occurrence of HL between 11-15 and 21-25 years respectively as well as a statistically significant difference between age and gender distribution. Olu-Eddo [13] reported 56 (6.8\%) cases of HL, most of which were mixed cellularity HL subtype (64.3\%) and lymphocyte depleted HL (19.6\%).

A fifteen-year epidemiological study of histopathological examination of lymphomas conducted in Ibadan showed that the lymphoma was identified in 51 males and 29 females between ages of 5 - 74 years and about $60 \%$ occurred in ages below 40 while modal group occurrence was in their thirties. Site of occurrence was frequent at a nodule and others occurring at several nodal sites, as intestine, liver, and spleen [12]. A case study in north east region of Nigeria evaluating lymphomas in 50 cases studied the patterns of presentations relative to sex, stated that 10 cases were of Hodgkin lymphoma with ratio 4:1 male and female respectively. Patterns of presentations revealed the following; 4 cases in stage II, 3, 2 and 1 were diagnosed to be I, III, and IV stages of HL respectively [14]. In Ile-Ife, of eighty-three histological samples of lymphoma cases, about $20.5 \%$ were identified as cHL, presenting frequently the cellular mixture subtype and eleven cases were observed to be associated with EpsteinBarr virus [15].

Kolawole [16] in a recent study on histological assessment of samples retrieved from different laboratories in Lagos, conducted in LUTH, indicated a sudden rise in incidence of HL. Thirty cases were identified as HL, two were marked as NLPHL, HL subtypes; NSHL, MCHL AND LRHL were estimated at $40 \%, 23.3 \%$, and $10 \%$, respectively above 7 years. This malignant disease displays a bimodal curve of its occurrence in more advanced nations. Also, the occurrence rate is said to be stable for over twenty years [17]. In the less advanced nations, the entire occurrence of Hodgkin lymphoma is lesser when compared to advanced nations excluding children below 15 years, where a higher occurrence is observed. Just a slight raised level of occurrence is observed throughout adolescence and young adulthood [4]. Generally the malignant neoplasm has been identified to persist more in males compared to females. In 2010, Worldwide it resulted in approximately 18,000 deaths downward from 19,000 deaths in 1990 [18]. Another recent study Uzoma, et al. [19] re-classified lymphomas cases from formalin fixed parafilm embedded tissues using WHO classification, and reports higher male to female ratio, ethnic variations in the lymphoma cases; CLL was prevalent in the Hausa compared to HL in the Yoruba ethnic while the Igbo ethnic group reported similar distribution of CLL, HL, and diffuse large B-cell lymphomas not otherwise specified (DLBCL, NOS). 


\section{Risk Factors and Symptoms of Hodgkin lymphoma}

The bimodal-curve occurrence of Hodgkin Lymphoma confirms the speculations that HL stems from two significantly different causes. These includes a causative agent with minimal infective activity which can be related to HL occurring in adolescence and/or a pathway related to other lymphomas which initiates tumorigenesis of the lymphoma in ages $>55$ years respectively [20]. Infection arising from Epstein Barr virus (EBV) is the most firmly established risk factor for developing HL. Approximately $1 / 3$ of patients in the western world with HL presents with undetected immune deficiencies [20,21]. These patients harbour EBV derived from the RNAs encoding the virus, nucleic acid antigen 1 and/or latent membrane protein (LMP1 and -2) in their tumor cells and are the probable driving causes of HL in these cases [22]. EBV is a lymphotropic virus of the gamma herpes family and spreads mainly via saliva. Research estimate $\leq 95 \%$ adults globally are Epstein Barr virus positive. Infection typically results in latent infection, but a proportion develops infectious mononucleosis (IM) which is associated with an elevated risk of developing HL [23]. Also the average time between IM and HL diagnosis is 4 years. Children $(<14$ yrs) as well as adults ( $>45$ yrs) have elevated risk factor(s) than young adults to harbor EBV positive tumor cells in HL. Genetic variation is a part of the risk factors in developing HL, the risk of Epstein-Barr virus positive Hodgkin Lymphoma is linked to genetic variants in the Human Leukocyte Antigen (HLA) class I region which also enhances risk in developing the lymphoma [24]. Patients who have been tested negative after clinical examination of EBV can however be observed frequently because the infective agent could be latent, hence undetected [25]. This process can be relevant as a determinant to clear off every doubt as well as identify higher risk of the lymphoma in individuals exposed to frequent blood products, assumed to be tested negative of HIV. Hence resulting in HIV false detection in such individuals which is proven as a significantly elevated risk in developing the lymphoma in all persons exposed [26-28]. In 1994, Hodgkin lymphoma was proven to be gotten from B-cells. However diverse underlying genetic factors can also raise the risk of Hodgkin lymphoma development indicating the aetiology is greatly multifaceted and may differ in different populations.

\section{Factors Contributing To Increasing Trends of Hodgkin Lymphoma in Nigeria}

Globally, about 37 million people are living with HIV infection, and Nigeria bears the second highest incidence of HIV infection globally and highest rate in sub-Saharan Africa with about 3.6 million persons living with the disease (NACA, 2017) [29]. Although there are declines in HIV prevalence in
Nigeria recently, this rate is still higher than rates in other African countries like South Africa and Zambia however these countries have higher adult HIV-infection rates of $19 \%$ and $11.5 \%$, respectively (NACA, 2017; UNAIDS, 2021) [29,30]. EBV is a common asymptomatic virus infecting over $90 \%$ population, however pathologic cases are very few $[10,31]$. EBV is associated with HL globally, it accounted for 40,109 (39.7\%) HL cases and 14,364 (44.1\%) HL-related deaths out of the total cases reported in 2017. This increasing incidence in the burden of EBV associated HL from 1990 to 2017 was higher in East Asia than in the African region [10]. However, in Nigeria, there are regional differences and cross-infections accounting for the burden of HL via EBV-HL, HIV-HL or EBVHIV infections $[19,32,33]$. A study from northern Nigeria showed over $50 \%$ EBV positivity in HL samples indicating it is an underlying factor for the incidence reported in these areas with lymphomas [33], another reported $60 \%$ EBV positivity [34], whereas a more recent study showed about $15.2 \%$ EBV-associated HL in the middle belt and southern regions [19], indicating regional differences in the prevalence. HIV also contributes to HL risk, studies showed the $7-12.5 \%$ HIV-associated HL in a Nigerian population [35,36], whereas another study reported 95.6\% EBV-HIV infection [32]. In Nigeria, lymphomas have been shown to be associated with higher cancer-related deaths especially in HIV-infected persons with 2.5times lower 2-year survival chance for (30\%) compared to HIV-uninfected persons (74\%). Late stage of lymphoma was also a contributing factor to the survival outcomes in HIV patients [36]. The identification of a precise prognosis and prediction biomarkers will allow affected individuals to get optimal treatments and lesser adverse effects of a high risk of death arising from the disease. Significant progress has been made in recent years in our understanding of the cellular origin of Hodgkin and Reed-Sternberg (HRS) cells in Hodgkin lymphoma. Some symptoms associated with HL include: swollen lymph nodes in the neck, armpits or groin region with no feeling of pain, reoccurring tiredness, fever, sweating at night, unexplained weight loss, severe itching and increased sensitivity to the effects of alcohol or pain in the lymph nodes after drinking alcohol.

\section{Mechanism of tumorigenesis in Hodgkin Lymphoma}

In Hodgkin Reed-Stenberg cells, there are multiple mechanisms involved (Figure 1) including repression of transcription factors in multiple signalling pathways of B-cell gene expression. Transcription factors such as NfkB (Nuclear factor Kappa B) and JAK/STAT (Janus Kinase/ Signal transducer and activator of transcription) pathway are implicated. The Basic components of $\mathrm{Nf}-\mathrm{kB}$ signalling pathway include:

- Receptors: (pro-inflammatory cytokines TNF $\alpha$, 
interleukin IL-1, bacteria LPS and many more.

- Signal adapter proteins: TNF receptor-associated factors (TRAFs) and receptor interacting proteins (RIPs).

- I Kappa B Kinase complex: (IKK) IKK $\alpha$ (IKK1), IKK $\beta$ (IKK2), regulatory subunit IKK $\gamma$ (NEMO),

- I Kappa B Proteins (IkB): IkB $\alpha, \operatorname{IkB} \beta, \mathrm{IkB} \varepsilon, \mathrm{Bcl}-3, \mathrm{p}-100$, p105

- NF-kappa B dimer/ protein: (p50, p52, p56) (ReIA), c-Rel, and ReIB.

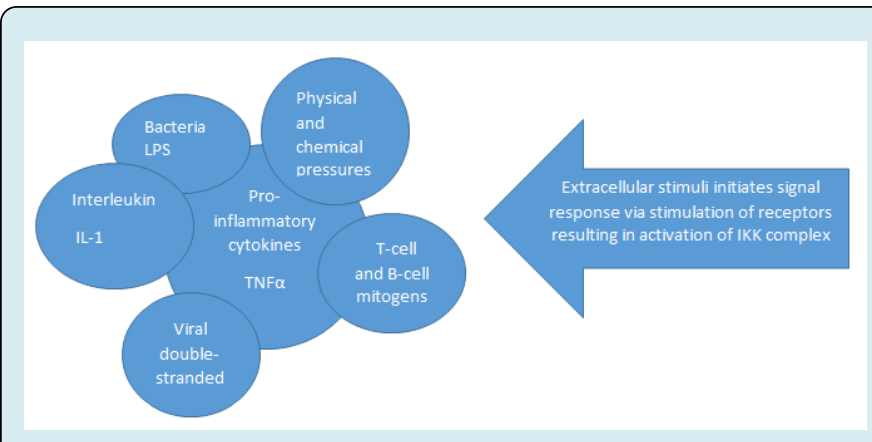

Figure 1: Extracellular stimuli involved in activation of I Kappa B Kinase complex in NF-KB pathways.

Canonical Pathway (NF-KB signalling pathway): When cells are subjected to various intracellular or extracellular stimuli (mainly extracellular stimuli initiates Nf-kB signalling pathway), receptors are signaled and activated resulting in a complex formed with signal adapter protein such as TRAFs and RIPs which leads to IKK complex induction. Hence IkB (NF-KB inhibitors) protein becomes phosphorylated and undergoes proteasomal degradation i.e. ubiquinated. The IkB protein is then degraded and NF-KB dimer is released. The NF-KB dimer is further activated by various posttranslational modifications and is translocated to the nucleus where they activate multiple genes, binds to the target gene and promotes the transcription of the target gene [37].

Alternative Pathway (NF-KB signalling pathway): Stimuli from EBV (LMP-1) infection up to $\geq 40 \%$, CD40 and BCMA signal receptors, thus activating the receptors which results in stimulating NIK (MAP3K14), and I Kappa B Kinase complex (IKK $\alpha$ ). However NIK is deregulated by TRAF3. The activated IKK complex results into p100 and further processed to p52 which is then translocated to the nucleus as a p52/ ReLA heterodimer. Research has shown that Hodgkin ReedStenberg cells activities is established in deregulating the canonical and alternative pathways respectively. Also diverse damage to nucleic acid (DNA), genetic mutations, and signals from LMP-1 activities of seropositive EBV of the lymphoma constitute great significance in the negative regulation of the pathways [37].

JAK/STAT pathway: This pathway is primarily initiated via cytokine signalling receptors. The cytokine receptors are activated via signals from STAT3, -5 , and -6 in HRS cells and are highly active. Also IL-13, -21 cytokine receptor are also activated. Negative regulation of this pathway is initiated either through increased amount of mutated JAK2 gene $[38,39]$ or its translocation into the nucleus as well as activities of mutated gene that often inactivate SOCS1 gene [40]. The occurrence of DNA damage and infective viral activities of the either in HRS cells is represented in percentage [37].

\section{Diagnosis and Current Treatment of Hodgkin Lymphoma}

Understanding the aetiology and biochemical mechanisms of pathogenesis of Hodgkin lymphoma would aid early detection of affected individuals who are highly prone to develop the lymphoma. Although the entire current diagnosis is good, however, a fraction of affected individuals will experience failed treatment, there are various founded risk-stratified therapies. A proper diagnosis of the physical state of patients, hematologic and biochemical diagnosis which includes red blood cell sedimentation rate, alkaline phosphatase, kidney and liver function test(s), $\mathrm{x}$ - ray, chest and abdominal computed- tomography scan, skeletal x-ray may be required. Individuals in clinical supra-diaphragmatic level one or two, who do not have beta signs indicate bone marrow is not likely involved. Hence marrow biopsy can be important in individuals with the beta signs and/or clinically advanced stages as well as and/or infra-diaphragmatic presentation also in individuals having bone injuries, pain, hypercalcaemic, and/or increased level of serum alkaline phosphatase [6]. However this marrow biopsy can be replaced later with [18] fluorodeoxyglucose-positron emission tomography and this is still being reviewed [41]. The sensitiveness of [18] fluorodeoxyglucose-positronemission tomography and computed- tomography is better than computed tomography alone to be able to identify both nodal and extra-nodal disease in prime stage [6]. Pathological identification of the lymphoma cells (Reed-Stenberg cells) is done microscopically using immunohistochemical staining for CD15 and CD30 markers which are expressed on the surface of the Reed-Sternberg cells in classical Hodgkin lymphoma. CD30 antibody-drug conjugate and immune checkpoint inhibitors have been approved for clinical management of HL, and these showed good efficacy [42]. PD-1 inhibitors (immunotherapy) targets programmed cell death proteins (PD-1) which are immune checkpoint ligands that regulate immune response. These have found usefulness in HL treatment. PD-1 protein is expressed on the surface of $\mathrm{B}$ and $\mathrm{T}$ cells, which are checkpoint in the cell cycle. PD-1 inhibitors activate the immune system to attack tumors. NF- $\kappa \mathrm{B}$ induces PD-L1 gene transcription by binding to its promoter, leading to up-regulation that promotes oncogenesis, inflammatory cytokines, and other cellular responses $[43,44]$. Hence inhibiting NF- $\kappa B$ signalling 
can possibly reduce PD-L1 expression and tumor growth. Natural compounds have demonstrated in cell lines and animal models to inhibit signalling pathways including PI3K/ Akt/mTOR pathway, and activation of NF- $\mathrm{KB}$ and STAT3 pathways which regulate proliferation, metastasis, apoptosis, angiogenesis. Several natural plants were shown to possess activities against lymphomas including HL in a review on natural compounds such curcumin, Japanese apricot, resveratrol, bromelain, Annonaceous acetogenins etc. [44]. Some early tumors might express signs and symptoms which can be observed, however it's not usually the situation. After cancer is diagnosed, the various stages of the cancerous growth will provide vital information concerning the extent of cancer in the body and likely response to treatment. Carrying out various medical examination provide indicators to identify HL in individuals who do not have symptoms.

Presently there are no broad screening examinations recommended for the malignancy due to no precise screening test identified to reduce the level of patients prone to death from Hodgkin lymphoma. The ideal process to identify Hodgkin lymphoma is to observe potential signs of which frequent symptom includes expansion or swelling of one or several lymph nodes, leading to a bump under the skin which doesn't hurt. It's seldom on the side of the neck, in the armpit or in the groin. Most times these swellings are caused by infections and not Hodgkin lymphoma, however bumps should be examined by doctors to identify the exact cause or origin. Cautious and constant diagnostics examinations should be done as it can identify individuals with risk factors for the disease which includes history of family. Such individuals usually do not come up with the lymphoma but the doctors and the individuals should be aware of signs. Individuals can have B-signs and symptoms which includes Fever with no infection, soaking night sweats, losing weight with no effort. These signs are vital to determine an individual physical state, other symptoms are skin itch, tiredness, no appetite, cough, breathing problems and pains in the chest.

\section{Conclusion and Recommendations}

Hodgkin lymphoma is an uncommon malignancy which involves the outlying lymph gland and affects several organs which includes liver, lung, and bone marrow. Hodgkin Reed Stenberg cells have been identified as the hallmark cell for the origin of this malignancy. Hodgkin lymphoma, however poses great threat to life as it is known to affect the immune system, resulting in individuals becoming more at risk to various infections and other diseases eventually leading to death. Therefore, more research and awareness should be done in identifying a unified genomic alteration as a pre-cursor of the pathogenesis of Hodgkin Lymphoma. Sensitization programs should be carried out to reduce high risk of developing the malignant neoplasm.

\section{References}

1. Bower M, Waxman J (2015) Lecture Notes: Oncology. John Wiley \& Sons.

2. Küppers R, Hansmann ML (2005) The Hodgkin and Reed/Sternberg cell. Int J Biochem Cell Biol 37(3): 511517.

3. Aggarwal P, Limaiem F (2019) Reed Sternberg Cells. In StatPearls. StatPearls Publishing.

4. Gobbi PG, Ferreri AJM, Ponzoni M, Levis A (2013a) Hodgkin lymphoma. Crit Rev Oncol Hematol 85(2): 216237.

5. Re D, Ku R, Diehl V (2005) Molecular Pathogenesis of Hodgkin's Lymphoma. J Clin Oncol 23(26): 6379-6386.

6. Gobbi PG, Ferreri AJM, Ponzoni M, Levis A (2013b) Hodgkin lymphoma. Crit Rev Oncol Hematol 85(2): 216237.

7. Carbone PP, Kaplan HS, Musshoff K, Smithers DW, Tubiana M (1971) Report of the committee on Hodgkin's disease staging classification. Cancer Res 31(11): 18601861.

8. Poppema S (2005) Immunobiology and pathophysiology of Hodgkin lymphomas. Hematology Am Soc Hematol Educ Program 2005(1): 231-238.

9. Salati M, Cesaretti M, Macchia M, El Mistiri M, Federico M (2014) Epidemiological overview of Hodgkin lymphoma across the mediterranean basin. Mediterranean Journal of Hematology and Infectious Diseases 6(1): e2014048.

10. Khan G, Fitzmaurice C, Naghavi M, Luai AA (2020) Global and regional incidence, mortality and disabilityadjusted life- years for Epstein- Barr virus- attributable malignancies, 1990-2017. British Medical Journal Open 10(8): e037505.

11. Ferlay J, Bray F, Soerjomataram I, Siegel RL, Torre LA, et al. (2018) Global cancer statistics 2018: GLOBOCAN estimates of incidence and mortality worldwide for 36 cancers in 185 countries. CA Cancer J Clin 68(6): 394424.

12. Oluwasola AO, Olaniyi JA, Otegbayo JA, Ogun GO, Akingbola TS, et al. (2011) A fifteen-year review of lymphomas in a Nigerian tertiary healthcare centre. J Health Popul Nutr 29(4): 310-316.

13. Olu Eddo AN, Omoti CE (2011) Hodgkin lymphoma: Clinicopathologic features in Benin City, Nigeria and update on its biology and classification. Nigerian Journal 
of Clinical Practice 14(4): 440-444.

14. Yakubu M, Ahmadu BU, Yerima TS, Simon P, Hezekiah IA, et al. (2015) Prevalence and clinical manifestation of lymphomas in North Eastern Nigeria. Indian J Cancer 52(4): 551-555.

15. Onwubuya IM, Adelusola KA, Durosinmi MA, Sabageh D, Ezike KN (2015) Lymphomas in ile-ife, Nigeria: Immunohistochemical characterization and detection of epstein-barr virus encoded RNA. J Clin Diagn Res 9(6): $-14-19$.

16. Kolawole H (2018) The Clinicopathologic Features of Hodgkin Lymphoma in Lagos, Nigeria: An Update on Biology and Histopathology Diagnosis. American Journal of Clinical Pathology 150(1): 102-103.

17. Townsend W, Linch D (2012) Hodgkin's lymphoma in adults. The Lancet 380(9844): 836-847.

18. Lozano R, Naghavi M, Foreman K, Lim S, Shibuya K, et al. (2012) Global and regional mortality from 235 causes of death for 20 age groups in 1990 and 2010: a systematic analysis for the Global Burden of Disease Study 2010. The Lancet 380(9859): 2095-2128.

19. Uzoma IC, Taiwo IA, Granai M, Di Stefano G, Sorrentino E, et al. (2021) Distinct pattern of lymphoid neoplasms characterizations according to the WHO classification (2016) and prevalence of associated Epstein-Barr virus infection in Nigeria population. Infectious Agents and Cancer 16(1): 36.

20. Hjalgrim H, Engels EA (2008) Infectious aetiology of Hodgkin and non-Hodgkin lymphomas: a review of the epidemiological evidence. J Intern Med 264(6): 537-548.

21. Armstrong AA, Alexander FE, Paes RP, Morad NA, Gallagher A, et al. (1993) Association of Epstein-Barr virus with pediatric Hodgkin's disease. Am J Pathol 142(6): 1683-1688.

22. Levine PH, Pallesen G, Ebbesen P, Harris N, Evans AS, et al. (1994) Evaluation of epstein-barr virus antibody patterns and detection of viral markers in the biopsies of patients with Hodgkin's disease. I J Cancer 59(1): 48-50.

23. Hjalgrim $\mathrm{H}$, Askling J, Rostgaard $\mathrm{K}$, Hamilton Dutoit S, Frisch M, et al. (2003) Characteristics of Hodgkin's lymphoma after infectious mononucleosis. N Engl J Med 349(14): 1324-1332.

24. Schwering I, Rajewsky K (2002) Symposium article Biology of Hodgkin's lymphoma. Annals of Oncology 13(1): 11-18.
25. Jarrett RF (2006) Viruses and lymphoma/leukaemia. The Journal of Pathology: A Journal of the Pathological Society of Great Britain and Ireland 208(2): 176-186.

26. Frisch M, Biggar RJ, Engels EA, Goedert JJ, Group ACMRS (2001). Association of cancer with AIDS-related immunosuppression in adults. JAMA 285(13): 17361745.

27. Clarke CA, Glaser SL (2001) Epidemiologic trends in HIVassociated lymphomas. Curr Opin Oncol 13(5): 354-359.

28. Dolcetti R, Boiocchi M, Gloghini A, Carbone A (2001) Pathogenetic and histogenetic features of HIV-associated Hodgkin's disease. Eur J Cancer 37(10): 1276-1287.

29. National Agency for the Control of AIDS (2021) National Strategic Framework on HIV and AIDS: 2017-2021. pp: 103.

30. UNAIDS (2021) Global HIV \& AIDS statistics-Fact sheet.

31. Young LS Yap LF, Murray PG (2016) Epstein- Barr virus: more than 50 years old and still providing surprises. Nat Rev Cancer 16(12): 789-802.

32. Okonko IO, Makinde TS, Okonko BJ, Ogbu O (2020) Immunological and epidemiological evaluation of EBV infections among HIV-1 infected individuals in Abakaliki, Nigeria supports the potential use of neutrophils as a marker of EBV in HIV disease progression and as useful markers of immune activation. J Immunoassay Immunochem 41(2): 158-170.

33. Iliyasu Y, Ayers LW, Liman AA, Waziri GD, Shehu SM (2014) Epstein-Barr Virus Association with Malignant Lymphoma Subgroups In Zaria, Nigeria. Niger J Surg Sci 23(1): 6-8.

34. Adelusola KA, Titiloye NA, Rotimi O, Durosinmi M (2009) Epstein Barr virus latent membrane protein-1 in Hodgkin's lymphoma in Nigerians. Afr Health Sci 9(3): 174-178.

35. Bolarinwa RA, Ndakotsu MA, Oyekunle AA, Salawu L, Akinola NO, et al. (2009) AIDS-related lymphomas in Nigeria. Braz J Infect Dis 13(5): 359-361.

36. Silas OA, Achenbach CJ, Hou L, Murphy RL, Egesie JO (2017) Outcome of HIV-associated lymphoma in a resource-limited setting of Jos, Nigeria. Infect Agent Cancer 12: 34.

37. Küppers R (2009) The biology of Hodgkin's lymphoma. Nat Rev Cancer 9(1): 15-27.

38. Joos S, Küpper M, Ohl S, von Bonin F, Mechtersheimer G, 
et al. (2000) Genomic imbalances including amplification of the tyrosine kinase gene JAK2 in CD30+ Hodgkin cells. Cancer Res 60(3): 549-552.

39. Van Roosbroeck K, Cox L, Tousseyn T, Lahortiga I, Gielen 0 , et al. (2011) JAK2 rearrangements, including the novel SEC31A-JAK2 fusion, are recurrent in classical Hodgkin lymphoma. Blood 117(15): 4056-4064.

40. Weniger MA, Melzner I, Menz CK, Wegener S, Bucur AJ, et al. (2006) Mutations of the tumor suppressor gene SOCS-1 in classical Hodgkin lymphoma are frequent and associated with nuclear phospho-STAT5 accumulation. Oncogene 25(18): 2679-2684.

41. Pakos EE, Fotopoulos AD, Ioannidis JPA (2005) 18F-FDG
PET for evaluation of bone marrow infiltration in staging of lymphoma: a meta-analysis. J Nucl Med 46(6): 958963.

42. Nishikori M (2019) Hodgkin lymphoma. Rinsho Ketsueki 60(5): 447-452.

43. Antonangeli F, Natalini A, Garassino MC, Sica A, Santoni A, et al. (2020) Regulation of PD-L1 Expression by NF- $\kappa B$ in Cancer. Front Immunol 11: 584626.

44. Batool A, Hazafa A, Ahmad S, Khan HA, Abideen HMZ, et al. (2021) Treatment of lymphomas via regulating the Signal transduction pathways by natural therapeutic approaches: A review. Leuk Res 104: 106554. 